\title{
Retinal Blood Vessels Extraction: Introduction and Future Trends
}

\author{
Khan Bahadar Khan ${ }^{1,2 *}$ and Amir A Khaliq ${ }^{1}$ \\ ${ }^{1}$ Department of Electronic Engineering, International Islamic University, Pakistan \\ ${ }^{2}$ Department of Computer Science, CECOS University, Pakistan
}

Submission: March 08, 2017; Published: April 26, 2017

*Corresponding author: Khan Bahadar Khan, Department of Electronic Engineering, International Islamic University, Islamabad, Computer Science Department, CECOS University, Peshawar, Pakistan, Tel: +92-333-5607397; Email: kb.khattak@gmail.com

\begin{abstract}
Retinal vascular structure is an imperative marker of numerous retinal and systemic maladies, which has persuaded the origination of different image segmentation strategies for the veins. The core target of this article is to distinctively introduce the open research issues in the retinal blood vessels segmentation and to discuss the similarities and dissimilarities between different approaches utilized to isolate blood vessels in retina images. The contemporary implications, the future strategies, and the open complications in automatic retinal vessels detection are also encapsulated.
\end{abstract}

Keywords: Review; Retina images; Blood vessels; Segmentation; Vasculature; Drive; Stare

\section{Introduction}

Segmentation play a key part in therapeutic imaging. Segmentation is utilized as a part of a many application, for example, investigation of physical structure, medical screening during evaluation of tortuosity, stenosis and angiogenesis [1]. In clinical analysis, segmentation assists the patients' to detect the level of the severity of the ailments. But, the aforesaid applications demand an adequate segmentation procedures that can isolate diverse sizes of the vessels as well as recognize irregularities in the vessels for better assessment. A portion of the accessible procedures are manual based. Manual isolation of vessel and non-vessel pixels is irksome, complex and time consuming, particularly during the investigation of enormous and composite databases when contrasted with computerized/ automatic segmentation [2]. In spite of the fact that the computerized procedures are deliberated to be precise and quick, despite everything they confront difficulties, for example, trouble in recognizing vessels from the non-vessels because of impediment created by blockage tissues, trouble in segmenting diverse widths of vessels particularly unhealthy vessels because of existence of artifacts in medical images, which leads to misclassification.

The vascular network of retina photograph contain the significant details which are utilized for the identification and exploration of different retinal disorders, for example, hypertension [3], glaucoma [4], and diabetes [5]. The eye's expert utilized fundus camera for capturing retinal photograph of the patients. These retinal photographs are used by the ophthalmologist for inspections, screening and analysis of various retinal disorders. The segmentation of blood vessels in retina images display significant vascular variations which are used for recognition and diagnoses of various ophthalmic abnormalities. The structure of vessel and non-vessel pixels is very homogenous in retinal images, which make vessels hard to isolate from the background pixels. Consequently, it is compulsory to utilize an appropriate image segmentation framework for precise extraction of retinal vasculature. These procedures depend on the image structures, for example, the cross-sectional profiles, identical intensity sections and boundaries [6].

Reviews and studies on the methodologies for extraction of vascular tree in medicinal images are present in the literature. Fraz et al. [7] categorize the retinal vessels extraction methods into seven groups based on the image processing techniques, namely, pattern recognition methods, mathematical morphology approaches, vessel tracking schemes, model based methods, parallel hardware based systems, multi-scale based procedures and matched filter based methodologies. Supervised and unsupervised approaches are in the sub-group of pattern 
recognition methods. Supervised schemes utilized already learned and trained data to choose whether a pixel belongs to a vessel or not, while unsupervised procedures achieve the vessel extraction with no earlier marked information. The word mathematical morphology is utilized as a tool for extracting image segments that are valuable in the demonstration and explanation of region shapes such as features, edges, skeletons and curved structures. Vessel tracking systems fragment a vessel between two points utilizing neighbourhood data and work at the level of a solitary vessel rather than the whole vascular network. The concept behind multi-scale frameworks for vasculature detection is to isolate facts associated with the blood vessel having variable size at multi scales. The computation time complexity of retinal vessel detection frameworks and requirements for real-time execution is resolved by parallel hardware based implementation of procedures. The matched filter based methodologies analyze the dissimilarities of the intensity level of the cross-section profile of the retinal image with the pre-set template or kernel.

\section{Discussion and Future Trends}

The target of this article is to discuss the open issues related to retinal blood vessels segmentation and to guide the scholars towards the interesting research directions. The recent vasculature segmentation methodologies still face trouble in isolating vessels due to image artifacts (such as intensity variations, noise, motion artifacts). A little number of available approaches are competent to detect vessels in medical images of different modalities. Researchers can further investigate to reduce the computation time, especially in supervised methods. A robust technique is required to segment blood vessels in healthy, unhealthy (disease infected) and noisy retinal images, to handle a large datasets containing images of different resolutions, to detect vessels of different widths, to locate vessels at their correct positions and to accurately compute vessels width. Another open area is to compute arteriolar-to-venular ratio (AVR) to isolate artery and veins. Complex preprocessing and postprocessing issue need to be addressed to decrease the computation time. Adaptive capabilities is required to control over-segmentation and under-segmentation under varying image conditions. The human intervention need to be eliminated for selection of region of interest, threshold selection and initial seed point selection.

\section{Conclusion}

The extraction of the retinal blood vessels has been a vigorously investigated zone in present age. The perfect localization of the retinal vasculature develops the foundation of numerous automated computer aided systems for analysis and detection of cardiovascular and ophthalmologic disorders. Even though many promising methods and strategies have been developed, there is still opportunity to get better in blood vessel extraction approaches.

\section{References}

1. Patton N, Aslam T, Macgillivray T, Pattie A, Deary IJ, et al. (2005) Retinal vascular image analysis as a potential screening tool for cerebrovascular disease: a rationale based on homology between cerebral and retinal microvasculatures. J Anat 206(4): 319-348.

2. Despotović I, Goossens B, Philips W (2015) MRI segmentation of the human brain: challenges, methods, and applications. Comput Math Methods Med 2015: 450341.

3. Mitchell P, Leung H, Wang JJ, Rochtchina E, Lee AJ, et al. (2005) Retinal vessel diameter and open-angle glaucoma: the Blue Mountains Eye Study. Ophthalmology 112(2): 245-250.

4. Leung H, Wang JJ, Rochtchina E, Wong TY, Klein R, et al. (2004) Impact of current and past blood pressure on retinal arteriolar diameter in an older population. J Hypertens 22(8): 1543-1549.

5. Staal J, Abràmoff MD, Niemeijer M, Viergever MA, van GB (2004) Ridgebased vessel segmentation in color images of the retina. IEEE Trans Med Imaging 23(4): 501-509.

6. Khan KB, Khaliq AA, Shahid M (2016) Correction: A Morphological Hessian Based Approach for Retinal Blood Vessels Segmentation and Denoising Using Region Based Otsu Thresholding. PloS one 11(9): e0162581.

7. Fraz MM, Remagnino P, Hoppe A, Uyyanonvara B, Rudnicka AR, et al. (2012) Blood vessel segmentation methodologies in retinal images-a survey. Comput Methods Programs Biomed 108(1): 407-433.
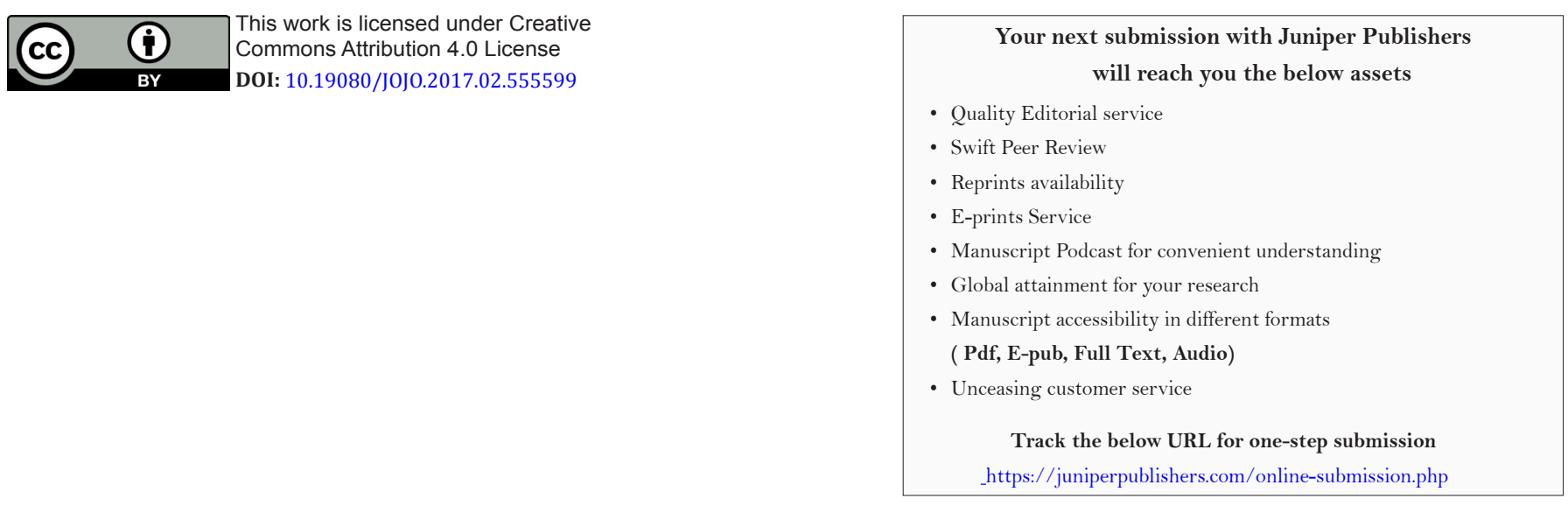